\title{
Limits of Spatial Resolution of a Raman Microscope
}

\author{
Fran Adar*, Sergey Mamedov* and Andrew Whitely*
}

*Horiba Jobin Yvon, 3880 Park Ave., Edison NJ 08820

The Raman microscope, a tool that was introduced in the mid 1970's is now a technique wellrecognized for its ability to characterize materials on the $\mu \mathrm{m}$ scale. It is finding usefulness in industrial manufacturing and in academic scientific disciplines of many sorts. When fully corrected optics are used in the tool, it can provide full resolution determined by the physical limits. The spatial extent of the laser focus is about $1 \mu \mathrm{m}$, a number that is derived from the formula for an Airy disc of light. In reality, the formula for the Airy disc,

$$
\omega_{0}=1.22 \lambda / \mathrm{na}
$$

predicts a focal spot equal to about $0.8 \mathrm{x}$ wavelength (the na is the numerical aperture of the optic). Actually this formula is not really accurate for a Gaussian laser beam being focused by a lens, but does provide a starting point for an estimate and/or predictor of spatial resolution. The formula for the depth of focus predicts a number somewhat larger because the na is taken to the second power.

These expressions do not predict the actual volume from which light is collected on a Raman microprobe. For a "perfect" sample, the focal volume of the laser would be expected to be a good predictor of the detected volume. But more often than not, the sample can be highly scattering, in which case the laser is not confined to the theoretical focal volume, and then the collection optics have a large role to play in the spatial resolution of the tool.

This paper will show results from empirical measurements determining spatial resolution from two types of sample. In the first case, the sample will be a pattern produced by lithographic techniques of the integrated circuit industry. Pattern features can be up to an order of magnitude smaller than a $\mu \mathrm{m}$. They have sharp interfaces and can be repetitive. We can measure the sharpness of the interface in the Raman map, and we can measure the modulation transfer function of a series of lines.

At the other extreme we will examine a highly scattering sample. The sample that we have chosen is a $\mathrm{Y}_{2} \mathrm{O}_{3}$-stabilized $\mathrm{ZrO} 2$ ceramic. The yttria stabilizes the zirconia in the normally high temperature tetragonal form, but stresses will produce cracked regions in which there has been a phase transformation to the normal room temperature monoclinic form. Because of the engineering uses for this material, there is interest in understanding the transformation, and in particular, in mapping the size of the transformation zone. It is not unusual for the ceramic particles to be of the order of a $\mu \mathrm{m}$ and every particle boundary has the potential to scatter light. Therefore the extent of the probed region is quite important to understand.

The first figure shows the result of a map of the lithographic sample. The pattern of vertical lines spaced $1 \mu \mathrm{m}$ apart is reproduced on the map. It is not know if bright spots on the map result from imperfections in the lithographic deposition or not. Figure 2 shows extracted profiles along an $\mathrm{X}$ row (left) and a Y column (right). On the left profile one can see that the intensity in the trough is well below the $50 \%$ level. On the right profile, one can see that the intensity of the horizontal silicon line goes from 20 to $80 \%$ in $0.5 \mu \mathrm{m}$. 
Figure 3 shows a micrograph of a stress-induced indent in $\mathrm{ZrO} 2$. The spectra on the right indicate the potential for discriminating between the two phases. The presentation will include Raman maps taken under autofocus conditions from which it will be possible to define empirically the spatial resolution.
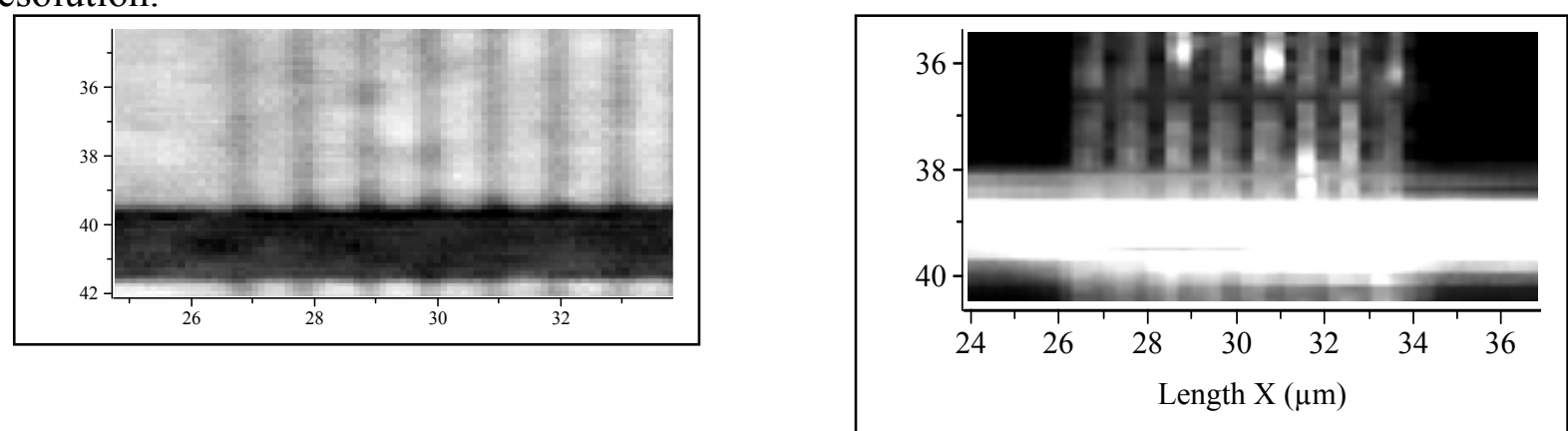

Fig. 1. The micrograph on the left shows a metallized pattern on a silicon wafer. The darker material is silicon. The vertical lines are $1 \mu \mathrm{m}$ apart, and are about $0.25 \mu \mathrm{m}$ wide. The micrograph on the right is actually a map of those lines produced by assigning intensity to the silicon signal.
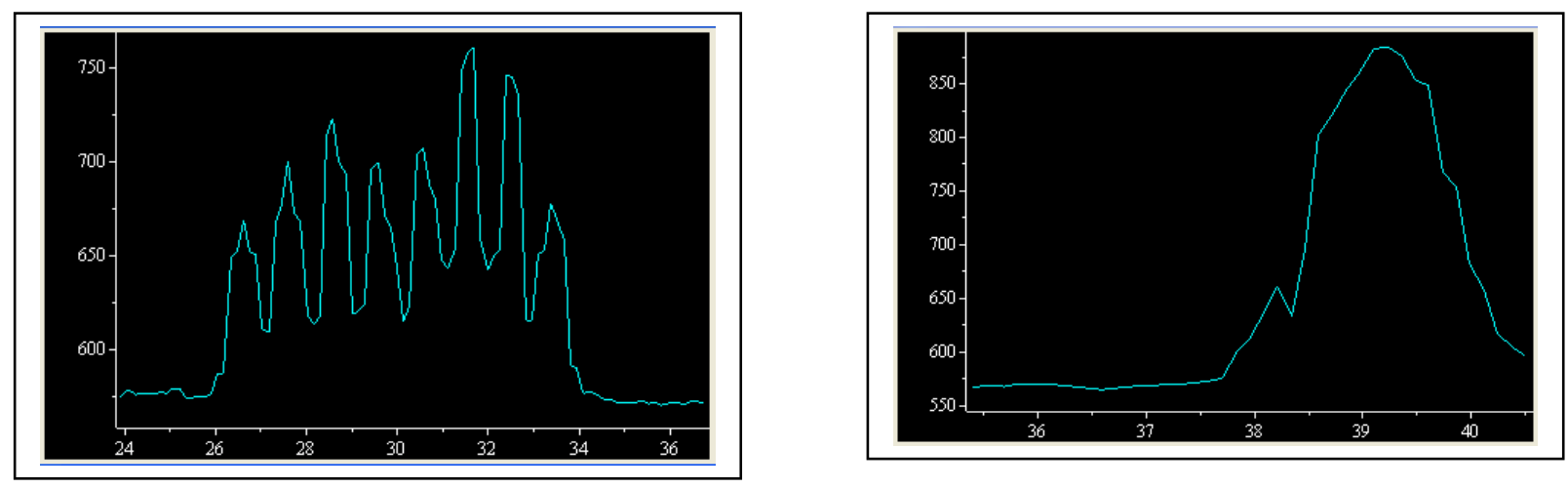

Fig. 2. Profiles extracted from the map on the right side of Fig. 1. The $\mathrm{X}$ profile on the left was extracted from line at $\mathrm{Y}=37.6 \mu \mathrm{m}$. The $\mathrm{Y}$ profile on the right was extracted from the column at $\mathrm{X}=37.7 \mu \mathrm{m}$.
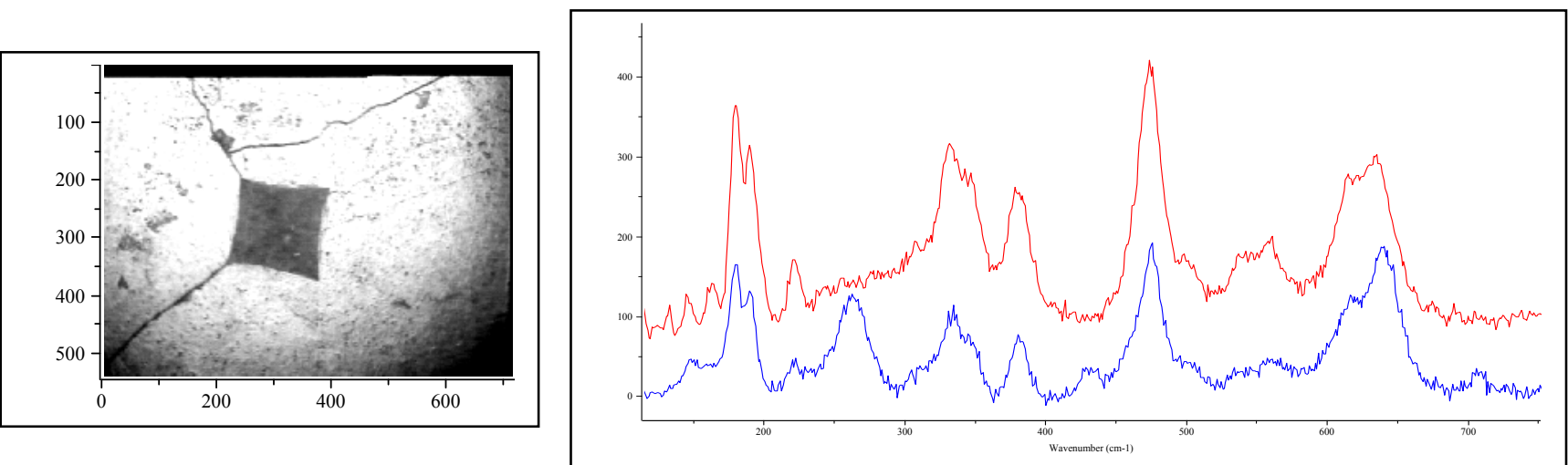

Fig.3. Optical micrograph of stress-1nduced indent $1 \mathrm{n} \mathrm{ZrO}_{2}$ (Ieft) and spectra of the monoclinic phase taken in the center of the indent (top) and the bulk alloy containing a mixture of tetragonal and monoclinic (bottom). 